\title{
Satellite Selection Methodology for Horizontal Navigation and Integrity Algorithms
}

\author{
Daniel Gerbeth, Ilaria Martini, Markus Rippl, Michael Felux, \\ German Aerospace Center (DLR)
}

\section{BIOGRAPHIES}

Daniel Gerbeth received a Bachelor and Master's degree in Electrical Engineering and Information Technology from Karlsruhe Institute of Technology, Germany in 2014. During Master studies he specialized in aerospace technology and navigation. After working in the field of sensor fusion and navigation aiding at Fraunhofer IOSB he joined German Aerospace Center (DLR) in May 2015 and is involved in the research on GBAS now.

Dr. Ilaria Martini received the Master's degree in telecommunication engineering and the Ph.D. in information technology from the University of Florence, Italy. She was at the Galileo Project Office of ESA/ESTEC in 2003, working on the performance of the Galileo Integrity Processing Facility. She was research associate in 2004 at the University of Florence and in 2005 at the the Federal Armed Forces Germany in Munich. In 2006 she joined the navigation department of Ifen $\mathrm{GmbH}$ in Munich. Since 2012 she works as research associate in the Institute of Communication and Navigation at the German Aerospace Center (DLR). Her main area of interest is GNSS Integrity Monitoring.

Markus Rippl received his diploma in electrical engineering and information technology from Technische Universitat Munchen in 2007. Since then, he has been a research associate with the Institute of Communications and Navigation at the German Aerospace Center in Oberpfaffenhofen near Munich, Germany. His areas of interest include GNSS integrity using receiver-side algorithms, next-generation RAIM algorithms and architectures, and the development of a future integrity architecture supporting ARAIM with an ISM.

Michael Felux received a diploma in Technical Mathematics from the Technische Universität München in 2009. The same year he joined the German Aerospace Center (DLR) where he has was working on the development of the GBAS GAST C testbed at the research airport in Braunschweig and its upgrade to GAST D. He was involved in flight testing and ground validation of the station and the approach procedures. Since 2015 he is coordinating DLRs research on GBAS-based navigation.

\begin{abstract}
With the new upcoming GNSS constellation in the future it might no longer be possible to use all satellites in view for navigation due to limited tracking channels. This is in particular true in the context of Advanced Receiver Autonomous Integrity Monitoring (ARAIM), where the use of dual frequency is favorable to mitigate ionospheric disturbances.

To address the issues of limited channels we propose two different satellites selection strategies adapted for Horizontal ARAIM in this paper. First a bare geometric approach which comes with almost no additional computation effort at the cost of less stable results. And second a heuristic optimization which improves selection results significantly while adding additional computational effort.

Both approaches are compared to brute force selected best sets in terms of resulting protection levels, computational cost and achieved ARAIM availability.

Results show the general applicability of both presented selection methods in Horizontal ARAIM. Using limited sets instead of all satellites in view can still provide global availability. Depending on the method more or less satellites are necessary to ensure sufficiently small and stable protection levels.
\end{abstract}

\section{INTRODUCTION}

In a few years, at least four GNSS constellations providing signals on multiple frequencies for civil users will be operational. This enhancement has drawn attention to further developing RAIM (Receiver Autonomous Integrity Monitoring) concepts that can make use of this multitude of available signals. ARAIM (Advanced Receiver Autonomous Integrity Monitoring), an integrity scheme that evolved from the Multiple Hypothesis Solution Separation (MHSS) algorithm [1], is a promising candidate for aviation users and other applications with high demand for integrity. It optimally exploits the massive measurement redundancy provided by two or more constellations, providing robustness against multiple satellite or constellation wide faults [2]. Current research in the field of ARAIM increasingly focuses on the trade space between different possible architectures to support ARAIM users with the dissemination of an Integrity Support Message (ISM) [3]. In light of a gradual increase, both on the number of operational satellites and on the operational history of the new constellations, ARAIM will first be devel- 
oped to allow horizontal navigation using the new signals and then extend its capabilities to allow also for vertical guidance in aircraft approach. ARAIM has been subject to many studies in the past years both in simulation [4] and using real measurements $[5,6]$. It becomes apparent from an implementation point of view that with a higher number of satellites, the computational complexity for a single epoch of ARAIM integrity increases drastically.

Satellite selection strategies can be one option to address this issue. Limiting the number of satellite channels and the number of supported constellations can potentially reduce the receiver complexity and save costs. Utilizing the selection from multiple constellations allows optimizing the performance per satellite, as the better distribution of more satellites in general allows better geometries. Performing all ARAIM user algorithms on a smaller number of satellites can then significantly reduce the necessary computational costs.

Satellite selection in general was topic of several papers in the past, providing different algorithms to perform the task. The common goal is in general to find a subset of all satellites currently in view which provides the best (whereby best depends on the application) navigation performance under given side conditions. Simple and computationally efficient approaches using only satellite elevation and azimuth were presented by Zhang [7], Song [8] and Park [9]. Also the widespread approach of selecting the highest satellites, assuming the best signal quality with highest elevation, falls into this category. Another group of algorithms tries to maximize polyhedron volumes or matrix determinants [10-12] as they correlate well with the geometrical dilution of precision (GDOP) of a set. Both of these strategies suffer from the missing possibility to take into account any satellite weighting (e.g. due to signal quality) and focus on 3D positioning, making them less suitable in our context. Phatak presented a method in [13] which allows efficient exchange of single satellites in a set which we will utilize later as well. Beyond that, approaches applying genetic algorithms [14] or artificial neural networks [15] were presented earlier.

While all these works show that satellite selection in general was covered from many perspectives within the last decades, ARAIM in particular has not been addressed so far. As ARAIM comes with several special characteristics, algorithms achieving good selections in terms of DOP are not necessarily suitable for ARAIM without changes. This is especially true for Horizontal ARAIM, focusing on the horizontal performance and therefore requiring appropriate geometries.

For this reason we adapt two multi constellation satellite selection algorithms particular for Horizontal ARAIM in this paper. After introducing the methodology they are assessed in terms of their resulting horizontal protection levels, computational load and achieved global availability for H-ARAIM.

\section{METHODS}

\section{ARAIM Basics}

ARAIM originates from Multiple Hypothesis Solution Separation [1] and is a RAIM method capable of obtaining navigation integrity for stand-alone users. It was designed to exploit the high number and quality of GNSS signals that will be available in the future: Multiple GNSS constellations, providing L1 and L5 signals that make dual frequency measurements also available for users of the aviation community. As opposed to classical weighted least square RAIM [16] it complies with more stringent integrity requirements that are mandatory for aircraft precision approach.

The most remarkable improvement is its ability to bound position errors (i.e. protection levels) under nominal, single and multiple fault conditions. It is expected that upon the availability of next-generation GNSS signals from GPS, Galileo, GLONASS and Beidou, the worldwide integrity performance using ARAIM will be sufficient to provide LPV-200 navigation capability without the need of conventional augmentation systems such as WAAS or EGNOS $[2,4]$. The standardization of ARAIM and the underlying assumptions have been discussed in $[17,18]$, and more recently further directed towards the definition of a suitable ground architecture for a light integrity support message (ISM) in [19, 20].

It is already foreseeable that together with the desirable leap in terms of navigation performance, the transition from RAIM to ARAIM will also increase complexity of the ground architecture and user algorithm [3].

ARAIM employs a multiple hypothesis approach to incorporate the potential effects of single or multiple satellite faults into its prediction of the worst case error, the Protection Level (PL). Any combination of faults is first evaluated with respect to its prior probability of occurrence. If a fault mode (i.e. one unique combination of faulted and healthy measurements) is likely to occur, a subset of measurements excluding the potential fault candidates is established and a subset based position is estimated. With all hypothetical position solutions merged into a union of position estimates it is possible to state the probability that the resulting interval contains at least one position solution based entirely on fault free measurements.

The remaining fault hypotheses which were not considered in the interval constitute the set of unmonitored hypotheses. The set of these hypotheses is chosen such that the sum of their probabilities is a fraction of the permitted integrity budget. For LPV-200 approaches, this overall integrity risk is defined as $P H M I=2 \cdot 10^{-7}$.

Because MHSS does not make a decision with respect to the fault states of the satellites involved in computing a position solution, every position solution with integrity contains reduced subset geometries. Thus this algorithm is much more susceptible to geometry size than classic RAIM [16], and even geometries with a few more satellites than the minimum (i.e., 5 measurements for two constellations), are often unsuitable to compute an error bound with ARAIM. On the other hand, too many ranging sources increase the 
computational complexity. The purpose of this work is therefore to find means to locate the 'sweet spot' when the number of available ranging sources is high compared with the number of necessary measurements.

The set of hypotheses taken into account for a specific geometry is derived from model parameters provided by the ISM (Integrity Support Message). Specifically, the parameters $p_{\text {sat }}$ and $p_{\text {const }}$ indicate the estimated probability that a single satellite, or a set of satellites within a constellation, may unknowingly be in a faulted state. For high values of $p_{\text {sat }}$, the hypotheses set grows and includes subsets of the visible geometry where more satellites have been excluded, i.e. smaller geometries. For a large $p_{\text {const }}$ value, hypotheses where a whole constellation is excluded must be computed. The relationship between $p_{\text {sat }} / p_{\text {const }}$, the geometry size of the all-in-view set and the hypothesis set size, is not linear because of the discrete allocation of probability into certain subsets. This effect has also an impact on the results shown hereafter.

For the horizontal case, the ARAIM protection level is computed for $q=(1,2)$ such that

$$
\begin{aligned}
& 2 Q\left(\frac{H P L_{q}-b_{q}^{(0)}}{\sigma_{q}^{(0)}}\right)+ \\
& \sum_{k=1}^{N_{\text {faul t modes }}} p_{\text {fault }, k} \cdot Q\left(\frac{H P L_{q}-T_{q, k}-b_{q}^{(k)}}{\sigma_{q}^{(k)}}\right) \\
& =\frac{1}{2} P H M I_{H O R}\left(1-\frac{P_{\text {sat, not mon. }}+P_{\text {const, not mon. }}}{P H M I_{V E R T}+P H M I_{H O R}}\right)
\end{aligned}
$$

This computation is an iterative process, where the allocation of the integrity budget into the $N$ hypotheses is optimized for the smallest possible HPL. We can see that the required probability for each of the hypotheses depends on the (horizontal) covariance of the corresponding subset geometry, $\sigma_{q}, q=(1,2)$, and on the bias contribution from the present measurements.

While the bias contribution is smaller for smaller subsets, the covariance grows as the geometry degrades. In particular with rather small geometries, there are often one or two satellites in the all-in-view set that are particularly important for the position solution, and their removal increases the solution covariance drastically. In the present work, we therefore try to find a computationally simple way to identify which satellites can be safely removed from the all-in-view set without a significant performance change.

\section{Fast Geometric Selection}

One approach presented here is inspired by previous work of Zhang et al. [7] and Song et al. [8]. While they select optimal sets in terms of GDOP we are primarily interested in horizontal performance and therefore HDOP. According adjustments are made to the algorithm.

From theoretical analysis can be seen that an optimal geometry in terms of HDOP consists of only a single satellite at $90^{\circ}$ elevation and all remaining satellites equally spaced (along azimuth) as low as possible. When considering ARAIM this

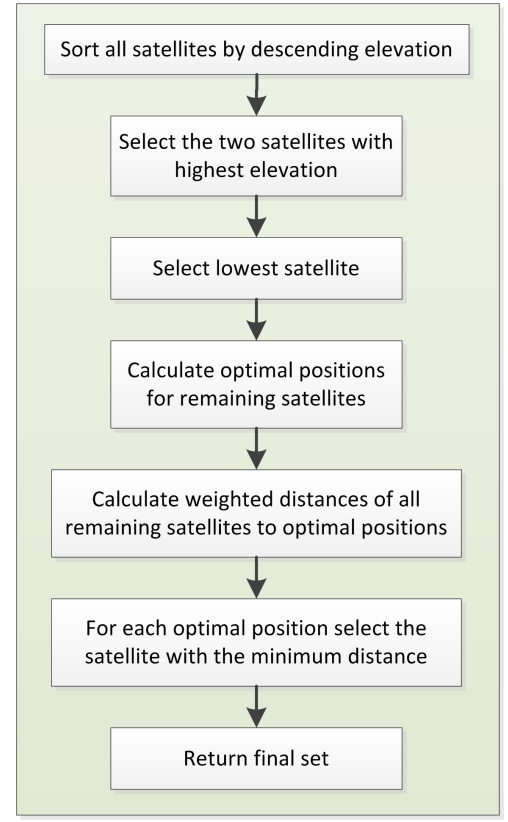

Figure 1: Schematic overview of geometric selection.

single satellite on top proved to be to few due to the fault modes. In terms of a satellite fault the remaining geometry with only low elevation satellites is mostly very weak, leading to large increases in the overall protection level as described before. To overcome this issue two high satellites are picked in any case, possibly leading to a higher HDOP but smaller average ARAIM protection levels. Apart from this modification the algorithm still tries to find low elevation satellites with a good distribution along azimuths. A schematic overview of the overall algorithm is given in Figure 1 and explained step by step in the following.

1. If less than $k=8$ satellites are selected the following steps are performed on each constellation separately. The returned set is the one with the best HDOP among them. Later these two options will be called $S C$ or $M C$ mode.

2. The algorithm starts with selecting the two satellites with the highest elevation.

3. Next, the lowest satellite is added to the selection.

4. Based on this satellite, optimal positions for the remaining $k-3$ satellites are calculated according to Equation 2. The azimuth of the lowest satellite, picked in step 3 is annotated with $a z_{0}$ here.

5. For each optimal position the weighted distances to all not yet selected satellites is calculated using the distance function in Equation 3.

6. Now the optimal positions calculated in step 4 are iterated and for each the not yet selected satellite with the smallest distance is added to the set.

7. The set of $k$ selected satellites is returned. 


$$
\begin{gathered}
a z_{\text {optimal }, i}=a z_{0}+i \cdot \frac{360}{k-2} \quad(\bmod 360), \\
e l_{\text {optimal }, i}=0 \quad i=1, \ldots, k-3 \\
\text { dist }=\alpha \cdot \Delta a z+(1-\alpha) \cdot \Delta e l^{\beta}
\end{gathered}
$$

The weighting factor $\alpha$ and $\beta$ in Equation 3 were determined based on an unconstrained optimization for 10,000 selections. Cost function in this optimization was the difference of the achieved HDOP of the geometric selection and the optimal selecting using brute force. Later in the simulations values of 0.95 for $\alpha$ and 2 for $\beta$ are used.

\section{Heuristic Optimization Selection}

The second selection method which is further studied in this paper is adapted from previous work we did on Ground Based Augmentation Systems. In [21] we presented a heuristic selection algorithm which uses information obtained by an all-in-view-solution to efficiently perform an iterative subset selection and optimization.

This method is based on the pseudoinverse $\mathbf{S}$ of the weighted geometry matrix $\mathbf{G}$ containing all available satellites:

$$
\mathbf{S}=\left(\mathbf{G}^{T} \mathbf{W G}\right)^{-1} \mathbf{G}^{T} \mathbf{W}
$$

where each row $\mathbf{G}_{i}$ of $\mathbf{G}$ is defined as

$\left[-\cos \left(E l_{i}\right) \cos \left(A z_{i}\right)-\cos \left(E l_{i}\right) \sin \left(A z_{i}\right)-\sin \left(E l_{i}\right) 10\right]$

for GPS or

$\left[-\cos \left(E l_{i}\right) \cos \left(A z_{i}\right)-\cos \left(E l_{i}\right) \sin \left(A z_{i}\right)-\sin \left(E l_{i}\right) \quad 0 \quad 1\right]$

in case of Galileo with $A z_{i}$ and $E l_{i}$ being the azimuth and elevation of the i-th satellite respectively. The weighting is performed in terms of the diagonal matrix $\mathbf{W}$ consisting of the $\sigma_{\text {mod }}$ values (see e.g. [3]) per satellite:

$$
\mathbf{W}=\left[\begin{array}{cccc}
\sigma_{m o d, 1}^{-2} & 0 & \cdots & 0 \\
0 & \sigma_{m o d, 2}^{-2} & \cdots & 0 \\
\vdots & \vdots & \ddots & \vdots \\
0 & 0 & \cdots & \sigma_{m o d, N}^{-2}
\end{array}\right]
$$

The measure which satellites get ordered by for the selection and optimization is derived from $\mathbf{S}$ as follows:

$$
\begin{aligned}
S_{\text {measure }, i}= & \sqrt{S_{1, i}^{2}+S_{2, i}^{2}} \\
& +\left(\alpha \cdot \log _{10} \frac{1}{p_{\text {sat }, i}}+\beta \cdot \log _{10} \frac{1}{p_{\text {const }, i}}\right)
\end{aligned}
$$

Only the along- and cross-track components in the $\mathbf{S}$ matrix are taken into account as we focus on horizontal navigation. The parameters $p_{\text {sat }}$ and $p_{\text {const }}$, representing the satellite and constellation fault probabilities, are taken from in the Integrity Support Message. They are included in the ranking in a way giving a satellite with a higher probability to be faulty a lower measure and therefore ranking it lower. The same happens for satellites part of a constellation with a higher fault probability. Weighting parameters $\alpha$ and $\beta$ are again found using optimization. The values used later on in this case are $\alpha=3.5$ and $\beta=0.3$.

This heuristic approach is adapted for ARAIM in two different ways. In both cases the general procedure is the same, only the minimization criterion during optimization differs. The first alternative uses actual ARAIM HPLs to assess the intermediate subsets during optimization. For every step during optimization the ARAIM HPL of the current set is evaluated, maximizing selection quality with the cost of a significantly higher computational effort.

In the second, performance optimized case there are no ARAIM protection level evaluations performed for the intermediate subsets. Instead 'pseudo protections levels' are used to assess the quality of the current selection. For a given subset the pseudo HPL is calculated as follows:

$$
\begin{aligned}
& \text { pseudoHPL }= \\
& \sum_{q=1,2}\left(\alpha \sqrt{\sum_{i} S_{q, i}^{2} \cdot \sigma_{\text {mod }, i}^{2}}+\beta \sum_{i}\left|S_{q, i}\right| \cdot b_{\max , i}\right)
\end{aligned}
$$

with $\alpha=0.5$ and $\beta=0.525$ again found by unconstrained optimization.

These pseudo protection levels take into account the relevant sigma and bias contributions of the satellites, but leave out all the fault mode evaluations and additional computations performed in the ARAIM user algorithm. The calculated values do not represent actual protections levels (hence pseudo) but correlate with the actual ARAIM HPLs and are therefore suitable for optimizing the selected set.

The core structure of the heuristic algorithm is represented in the scheme in Figure 2 and explained step by step in the following.

1. In case the number of satellites to select is smaller than 8 , a selection will be performed for each constellation separately (SC-mode) and the best of these will be used. This considers the fact that sets with less than 4 satellites per constellation tend to have larger protection levels, as no subset solutions within the constellations can be calculated. Using only satellites of a single constellation leads to better protection levels even with a weaker geometry in most of this cases.

2. Using all satellites in view the $\mathbf{S}$ matrix and the above mentioned $S_{\text {measure }}$ are calculated.

3. The satellites are ordered descending by their $S_{\text {measure }}$ and the first $k$ satellites are selected as first candidate set.

4. Starting with this set the optimization loop proceeds. At first the $S_{\text {measure }}$ is calculated within the satellite subset from the previous step.

5. Beginning with the last satellite in the ranking within the set, exchanges with the remaining (not selected) 


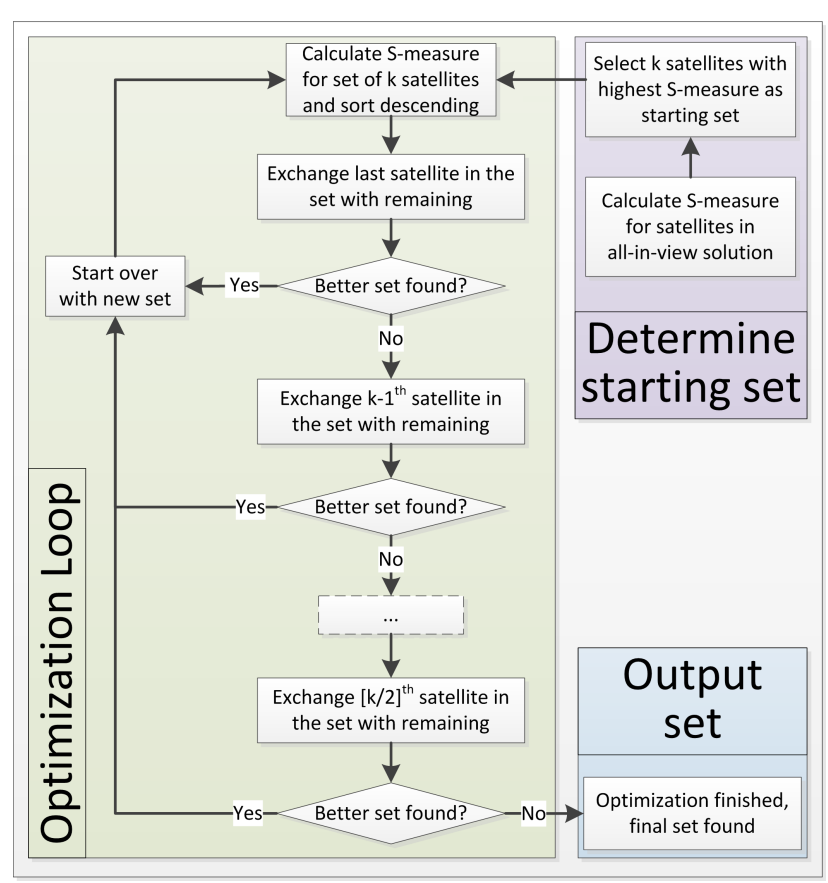

Figure 2: Schematic overview of heuristic selection.

satellites are performed. The remaining satellites are hereby iterated in descending order of their all-in-view $S_{\text {measure }}$. In other word satellites with a higher probability of being part of a favorable set are tried first.

6. For each set with the single satellite exchanged the optimization criterion (ARAIM HPL or pseudo HPL) is calculated. Instead of recalculating the complete $\mathbf{S}$ matrix the revolving door method from Phatak [13] can be utilized here to significantly speed up the process, especially in case of the pseudo HPL heuristic.

7. Whenever a better set was found (smaller HPL) the steps from 4 on repeat again.

8. If no exchange of the last satellite led to an improvement, the last but one satellite is exchanged next.

9. This continues until the second half of the satellites was checked. In case no improvement was found anymore, the optimization is finished.

The termination point of the algorithm (at which satellite the optimization stops) is a tuning parameters which allows to decide between computational cost and selection quality. Everything from optimizing up to the first satellite to performing no optimization and just using the first candidate set (end of step 3) is an option here. Depending on the necessary performance and available computational power one can choose an appropriate optimization depth here.

What we have seen is that most optimization potential lies within the last third of the satellite Thus, as a trade-off between computation time and selection quality we chose to optimize up to $\lfloor k / 2\rfloor$ in our simulations.

Apart from optimizing till a certain satellite one could also think of other stopping criteria, like the achieved (pseudo)

\begin{tabular}{c|c}
$P_{\text {sat }}$ & $10^{-5}$ \\
\hline$P_{\text {const }}$ & $10^{-8}$ \\
\hline URA & $2.5 \mathrm{~m}$ \\
\hline URE & $1.667 \mathrm{~m}$ \\
\hline Elevation limit & $5^{\circ}$ \\
\hline$b_{\text {nom }}$ & $000] \mathrm{m}$ \\
\hline$b_{\text {max }}$ & {$[0.750 .750 .75] \mathrm{m}$} \\
\hline PHMI $_{\text {HORZ }}$ & $9.8 \cdot 10^{-8}$ \\
\hline PHMI $_{\text {VERT }}$ & $2 \cdot 10^{-9}$ \\
\hline HAL & $40 \mathrm{~m}^{-6}$ \\
\hline$P_{\text {FA_HOR }}$ & $3.9 \cdot 10^{-6}$ \\
\hline$P_{\text {FA_VERT }}$ & $9 \cdot 10^{-8}$
\end{tabular}

Table 1: Overview of simulation parameters (equal for both GPS and Galileo).

protection level. As soon as a set is found which falls below the alert limit with a certain margin, the optimization could be stopped immediately.

\section{RESULTS AND DISCUSSION}

\section{General Setup}

All ARAIM HPLs in this work are calculated using the MAAST for ARAIM environment [22, 23]. Nominal almanacs with 30 (GPS) and 27 (Galileo) satellites were used for the simulations. The simulated location (except for the global availability) was Munich Airport $\left(48^{\circ} 21^{\prime} \mathrm{N}, 11^{\circ} 47^{\prime} \mathrm{E}\right)$.

Relevant ARAIM parameters were chosen according to [3] and are gathered in Table 1.

For HDOP and HPL comparisons 1000 satellite configurations over 10 days using the mentioned almanacs were generated. As simulation hardware an Intel Core i7-4600U CPU @ 2.10 GHz with 8GB RAM was used and all simulations were performed within MATLAB 2016a.

\section{Minimum DOP Selection}

At first we will take a look at the selection performance only in terms of geometry. The measure to compare the different selection strategies in this regard is the horizontal dilution of precision as we focus on horizontal navigation. HDOP gives an indication how an error in the range domain projects into the horizontal position error. Accordingly smaller values, projection factors, are better. As the DOPs are dimensionless, the results in the following plots are always factors representing the increase compared to the optimal HDOP achieved using brute force search.

In Figure 4 we start with the geometric selection. We can observe an exponentially improving average performance with median values well below a factor of 2 from 5 satellites on. Starting from 10 satellites on also most of the outliers are below this value. For fewer satellites the spread of the results starts growing significantly, making the method less reliable for small sets. 


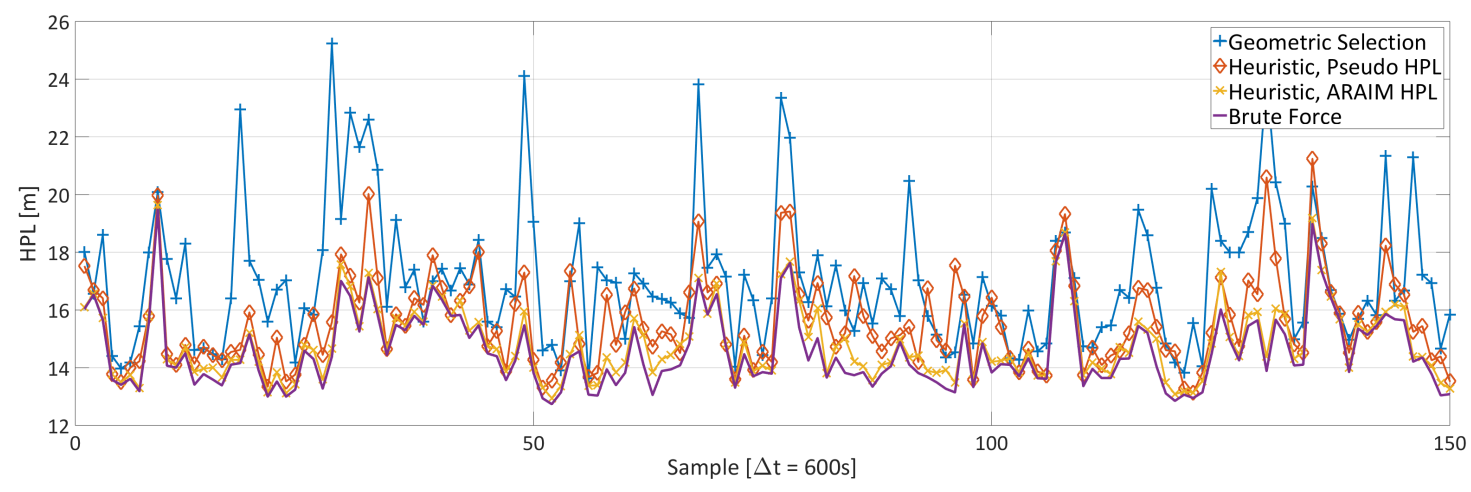

Figure 3: Exemplary comparison of HPLs over time for different methods selecting 12 satellites.

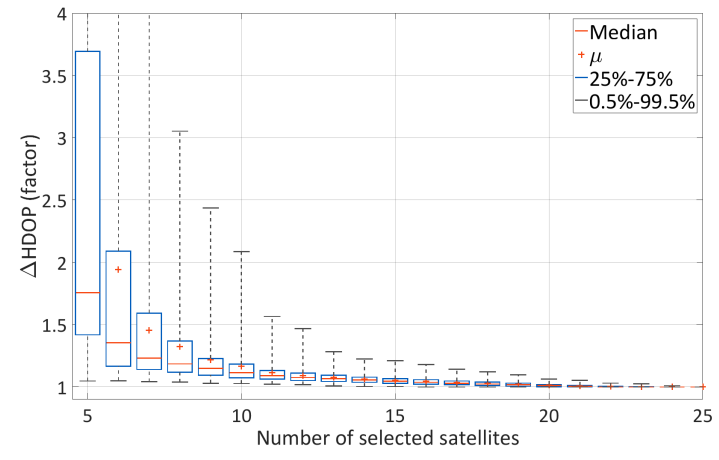

Figure 4: Geometric selection: deterioration of HDOPs compared to optimal subset for different set sizes.

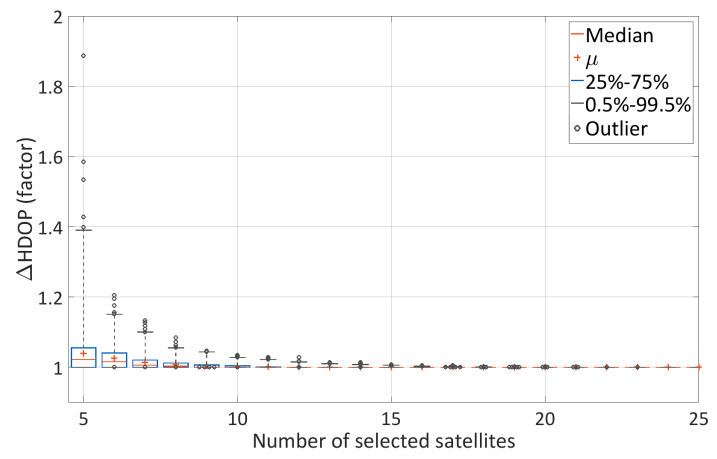

Figure 5: Heuristic selection: deterioration of HDOPs compared to optimal subset for different set sizes.

Figure 5 in comparison shows the performance of the heuristic selection. In contrast to the descriptions in Section II. the optimization is now performed in terms of HDOP (instead of ARAIM HPL/pseudoHPL). Except for this, the algorithm stays unchanged. Clearly visible is the much more stable performance with even the worst outlier during the simulation staying below a factor of 2. Median and mean values stay well below 1.05 starting from 5 satellites already which shows the stable performance of the selection heuristic under all circumstances.

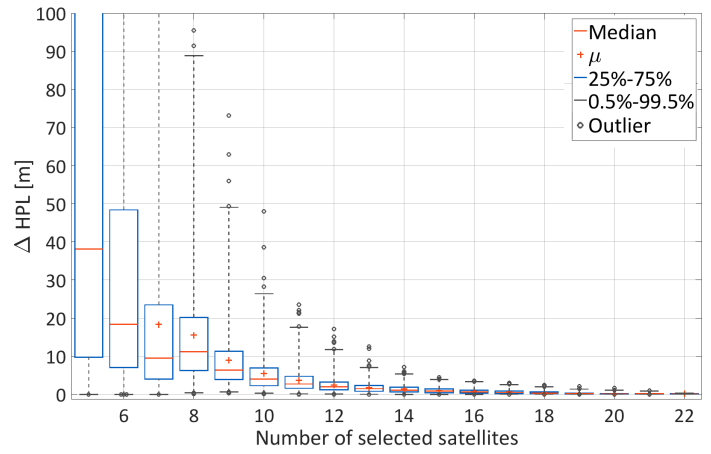

Figure 6: Geometric selection: deterioration of HPLs compared to optimal subsets for different set sizes.

\section{Minimum HPL Selection with Fixed ISM}

After assessing the performance in terms of sheer geometry we now look at actual ARAIM protection levels. For the simulations in this part a fixed ISM was assumed with the parameters given in the general setup section.

We start off with an exemplary protection level plot over time to give an idea of the absolute protection levels achieved and make the following relative results more meaningful. Figure 3 shows the achieved HPLs for the different selection strategies when selecting 12 satellites from the simulated GPS+Galileo scenario. Shown are the results over 25 hours. As we can see the optimal protection levels vary between 13 and 20 meters over the day. The heuristic approaches follow the brute force optimal selection very well for the whole time, especially the ARAIM HPL heuristic. Few situations, e.g. around sample 75 and 130, can be found where the pseudoHPL heuristic shows significant spikes. The errors reach up the $7 \mathrm{~m}$ here, starting from a protection level of about $14 \mathrm{~m}$. In most of this situations a major increase can also be seen in the geometric approach. This indicates the similarity of these approaches, optimizing the selection (mainly) geometric. In most of the samples a geometrically strong subset might have been found, but with unfavorable properties in terms of ARAIM, leading to a largely increased HPL.

After showing the performance of the selection over time, we take a look at the statistics in the following. Keeping the 


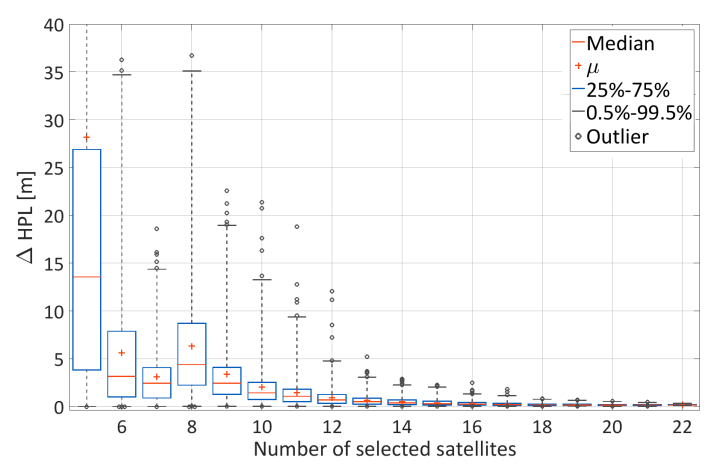

Figure 7: Heuristic selection using pseudoHPL: deterioration of HPLs compared to optimal subsets for different set sizes.

same order as before we start with the geometric selection again. Figure 6 shows the difference of the protection levels to an optimal brute force selection for set sizes from 5 to 22 satellites. The behavior from Figure 4 can be found again, even more pronounced. This makes sense as to the existing challenge of finding a good geometry comes the issue that especially for small set sizes the ARAIM protection level does not always coincide with a favorable DOP. When a single satellite dominates the performance or the distribution of satellites to the constellations is unfavorable, a set might have a good DOP but still result in a high protection level. This is mainly due to satellite and constellation faults which are taken into account during the ARAIM protection level calculation. To partly overcome the latter of the two issues the same SC/MC-selection-mode is introduced here as in the heuristics. Limiting sets smaller than 8 satellites to GPS or Galileo only, prevents selections which provide a standalone solution for neither of the two systems. This shows in the comparably small performance decrease from 8 to 7 satellites.

When we turn to the heuristic we see again the significantly less fluctuations for fewer satellites. In Figure 7 the statistics for the increase in HPL are given for the heuristic using pseudo protections levels as optimization criterion. From 12 satellites on the performance shows very stable with deltas below $5 \mathrm{~m}$ in almost all cases. Below this set size especially the occasional outliers start to increase significantly, making the selection less reliable, but on average still viable. The jump between 7 and 8 satellites results, as before, from the switch from SC to MC selection. In the case of 8 satellites an additional gain can be achieved by checking the $\mathrm{MC}$ as well as the SC selection results and using the best of the three.

\section{Minimum HPL Selection with Random ISM}

This section aims to give a hint what happens to the selection performance when the assumption of equal fault probabilities for all satellites and constellations as well as equal biases is no longer valid. To show a worst possible case we created a simulation with the mentioned parameters randomly chosen from the valid values defined in [3]. In Figure 9

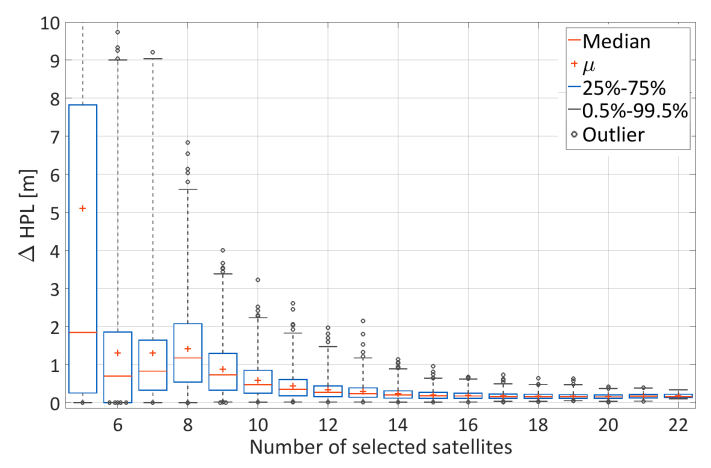

Figure 8: Heuristic selection using real HPL: deterioration of HPLs compared to optimal subsets for different set sizes.

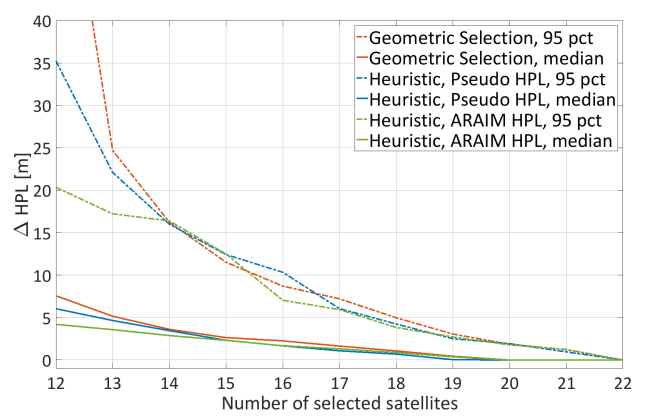

Figure 9: Performance comparison (median and $95^{\text {th }}$ percentiles) of the studied methods when using random ISM parameters.

we compare median and $95^{t h}$ percentiles of the different approaches with the optimal selection for 12 to 22 satellites. Again the above mentioned full dual constellation scenario is the basis for the geometries. The protection levels in general are - as expectable - higher and more fluctuating than before due to the different satellite and constellation fault probabilities. For all selection methods a major increase of the HPL difference can be recognized compared to the 'simple' case of uniform parameters. Also are the different algorithms closer in terms of their performance while mostly showing the same ranking among them.

\section{Global Availability}

After comparing the performance of the different algorithms statistically for a single location another assessment in terms of global performance shall be given. For this reason global simulations are performed to show the expected availability and average achieved horizontal protection levels for different scenarios. All simulations are based on a 10 by 10 degrees grid using the same almanacs as before. In total 10 days are simulated with a sampling of 600 seconds.

Figure 10 shows exemplary the $99.5 \%$ horizontal protections levels when selecting 12 satellites using the pseudo HPL heuristic. No significant influence of the location on the selection algorithm is present as the performance is quite stable along longitudes and shows the typically better performance close to the equator along latitudes. Globally 


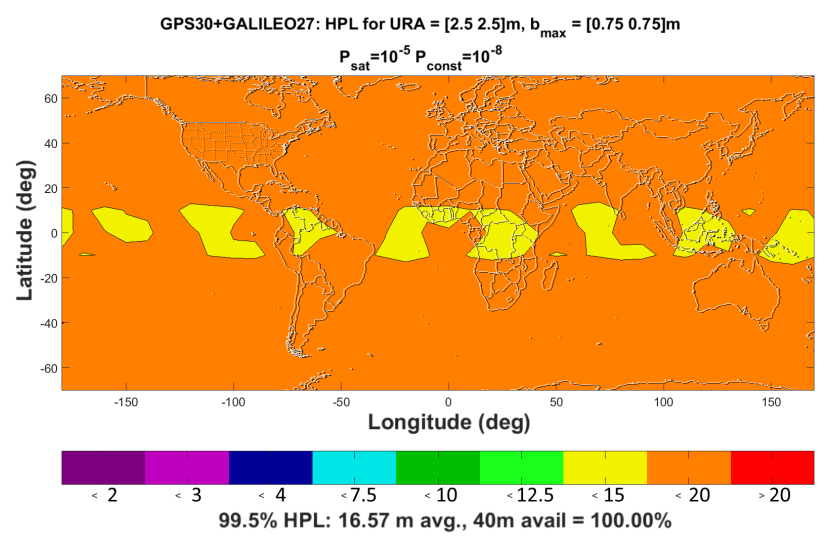

Figure 10: Global HPLs heuristically selecting 12 satellites.

\begin{tabular}{c|c|c} 
Method & $\mathbf{9 9 . 5 \%}$ HPL avg. & $\mathbf{4 0} \mathbf{~ m}$ avail. \\
\hline Full dual constellation & $13.16 \mathrm{~m}$ & $100 \%$ \\
\hline Heuristic real HPL, 12 & $14.73 \mathrm{~m}$ & $100 \%$ \\
\hline Heuristic pseudo HPL, 8 & $37.24 \mathrm{~m}$ & $99.8 \%$ \\
\hline Heuristic pseudo HPL, 10 & $21.55 \mathrm{~m}$ & $100 \%$ \\
\hline Heuristic pseudo HPL, 12 & $16.57 \mathrm{~m}$ & $100 \%$ \\
\hline Heuristic pseudo HPL, 14 & $14.35 \mathrm{~m}$ & $100 \%$ \\
\hline Geometric, 12 & $30.16 \mathrm{~m}$ & $99.99 \%$
\end{tabular}

Table 2: Average protection levels and availability for various other selection modes.

the $99.5 \%$ HPLs stay below 20 meters with an average of $16.57 \mathrm{~m}$ and therefore well below the alert limit of $40 \mathrm{~m}$ in case of Horizontal ARAIM. Thus $100 \%$ availability are achieved during the simulated scenario.

In Table 2 we additionally show simulation results for various other selection scenarios as well as the all-in-view case as reference. Selecting 10 instead of 12 satellite with the pseudo HPL heuristic still ensures $100 \%$ availability but already leads to significantly larger average HPLs. Going even further to 8 satellites increases the $99.5 \%$ HPL average almost to the alert limit, no longer proving full availability. This indicates a limit of 8-10 satellites when using this selection in terms of a multi-constellation scenario.

Looking at the results for the geometric selection reveals problems even in case of 12 satellites. Even though the availability is approximately $100 \%$, the average $99.5 \%$ HPL is already $30 \mathrm{~m}$ and therefore almost twice as high compared to the heuristic. When using this method at least more than 12 satellites need to be selected to ensure a stable performance without availability issues due to occasional unfavorable geometries.

In terms of the heuristic method using real ARAIM HPLs a significant improvement of almost $2 \mathrm{~m}$ to the pseudo HPL can be found. With an average 99.5\% HPL of $14.73 \mathrm{~m}$ the difference to the all in view solution shrinks to less than $2 \mathrm{~m}$. This shows also the minor protection level contribution of many satellites in the dual constellation case. Each additional satellite has a shrinking influence on the performance, rendering the advantage of more than 12 or 14 satellites comparably small.

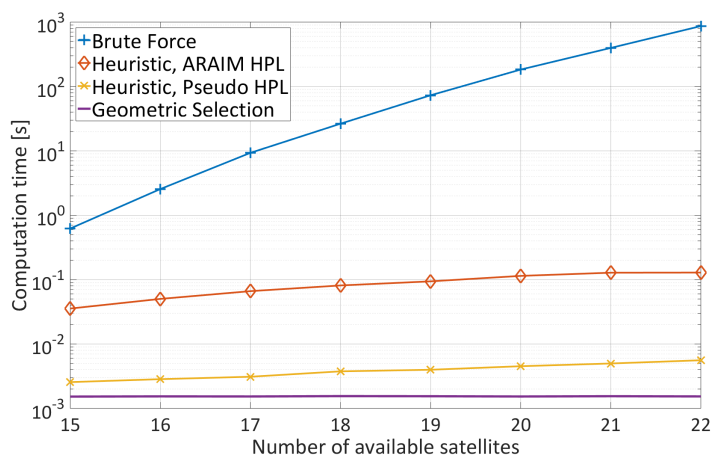

Figure 11: Comparison of average computation time per selection when selecting 12 satellites out of 15 to 22 available satellites.

\section{Computational Load}

In this section we finally compare the computational load of the proposed algorithms as well as an optimal brute force selection. The general results can be found as expected - the most simple geometric algorithm performing no optimization loops or similar is fastest in any case, followed by the pseudoHPL heuristic, the heuristic using real HPLs and the brute force search.

In Figure 11 the scaling of the computation time in terms of available satellites to choose from is shown. During the 10 days simulations time 15 to 22 satellites are visible at the specified location (Munich Airport). From these a fixed number of 12 satellites was selected using the different approaches. The average time per selection was computed based on this using MATLAB as simulation and timing environment and the hardware mentioned in the general setup in Section III.

Fastest is expectably the geometric approach which shows basically no dependency on the number of available satellites, as the selection procedure is not affected. The heuristic approach using pseudo protection levels is about 2 to 4 times slower and scales slightly when more satellites are available. Using real ARAIM HPLs in the optimization process leads to an expected almost constant offset in the range of a factor of 20. This can be explained with the additional complexity necessary to compute ARAIM HPLs instead of pseudo HPLs, while the amount of necessary optimization loops stays comparable. Finally, the brute force computation time scales heavily with the number of visible satellites, starting about one order of magnitude slower than the heuristic and ending up at almost four.

Figure 12 shows the scaling of the computation time in terms of the number of selected satellites. From the above described 1000 satellite configurations over 10 days at first all situations were chosen, when exactly 20 satellites were visible (above the $5^{\circ}$ elevation mask). For this cases (390 in total) the selection of 5 up to 19 satellites was performed using the different approaches.

The results of brute force selection follow the expected complexity of choosing $k$ out of $N$ with a maximum when 


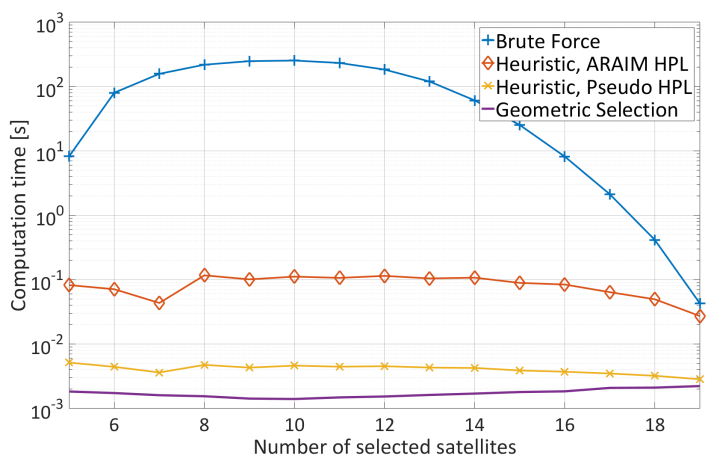

Figure 12: Comparison of average computation time per selection when selecting 5 to 19 satellites out of 20 available satellites.

selecting half the number of available satellites. For 20 satellites this grows already until up to 4 minutes for performing a single selection and spans over almost 4 orders of magnitude. The geometric method shows only minor dependency on the number of selected satellites, staying between 1.4 and $2.2 \mathrm{~ms}$ per selection.

Comparing the two variants of the heuristic as before the same almost constant offset between them can be seen. Both approaches show only minor dependency on the number of selected satellites and hence the bare combinatorial complexity of the problem. This leads to a particularly better performance compared to brute force in relevant regions, selecting for example 10-14 satellites in a dual constellation scenario. The necessary time is almost constant between 8 and 14 satellites and slowly shrinks afterwards. What can be seen from 5 to 7 satellites is the effect of the $S C / M C$ mode change. Basically two selections from 8-12 satellites are in the end less computational costly than a single selection from the combined set of satellites. This leads to the jump going up to 8 satellites where the selection is again performed on GPS and Galileo combined.

\section{CONCLUSIONS}

In this work we have shown the applicability of adapted selection methods to horizontal ARAIM. A non-iterative geometric approach showed to be feasible under certain circumstances while adding almost no additional computational effort. Nevertheless, leaving out all the implications of subset faults etc. in ARAIM can not guarantee favorable selections in all situations. Especially for very small sets the performance fluctuates strongly, making the approach less suitable for safety critical applications.

Performing an iterative optimization on the other hand achieves much more stable results throughout different satellite configurations. Evaluating the subsets with pseudo protections levels for performance maximization leads to drastically reduced computational load but also comes with a deteriorated performance. This is again especially the case when selecting small subsets where it is more likely that a strong geometry not necessarily coincides with a favorable ARAIM protection level.
Best and most stable selection performance and only minor changes on global average HPL are achieved using the heuristic which evaluates real ARAIM HPLs. In the performed simulations the necessary time for a single selection from up to 22 satellites stayed well below a second at all times. This might still be feasible depending on the application. On the other hand, the time to calculate an ARAIM protection level depends strongly on the convergence of involved algorithms This makes the necessary time for the selection harder to predict which could bring additional issues in an operational context.

Global simulations showed the heuristic using pseudo protection levels as a good trade-off in terms of performance and required computation time. Selecting 10 satellites already provides $100 \%$ global availability in the nominal dual constellation case. For 12 satellites the average global HPLs increase only $25 \%$ compared to the all-in-view solution which is only $13 \%$ worse than the selection using real HPL heuristic. 


\section{References}

[1] Boris S. Pervan, Samuel P. Pullen, and Jock R. Christie. "A Multiple Hypothesis Approach to Satellite Navigation Integrity". In: Journal of The Institute of Navigation 45.1 (1998).

[2] Juan A. Blanch et al. "Baseline Advanced RAIM User Algorithm and Possible Improvements". In: IEEE Transactions on Aerospace and Electronic Systems 51.1 (2015).

[3] Working Group C ARAIM Technical Subgroup. Milestone 3 Report. Tech. rep. 2016. URL: http: / / ec . europa . eu / growth / tools-databases / newsroom / cf / itemdetail. cfm? item_ $i d=8690$

[4] Markus Rippl, Alexandru Spletter, and Christoph Günther. "Parametric Performance Study of Advanced Receiver Autonomous Integrity Monitoring (ARAIM) for Combined GNSS Constellations". In: Proceedings of the 2011 International Technical Meeting of the Institute of Navigation. 2011.

[5] Markus Rippl. "Real Time Advanced Receiver Autonomous Integrity Monitoring in DLR's MultiAntenna GNSS Receiver". In: Proceedings of the 2012 International Technical Meeting of the Institue of Navigation. 2012.

[6] Markus Rippl et al. "ARAIM Operational Performance Tested in Flight”. In: Proceedings of the 2014 International Technical Meeting of The Institute of Navigation, ITM2014. 2014.

[7] Miaoyan Zhang and Jun Zhang. "A Fast Satellite Selection Algorithm: Beyond Four Satellites". In: IEEE Journal of Selected Topics in Signal Processing 3.5 (Oct. 2009), p. 8. ISSN: 1932-4553. DOI: $10.1109 /$ jstsp . 2009 . 2028381. URL: http : / / dx . doi.org/10.1109/JSTSP. 2009.2028381.

[8] Dan Song et al. "An Algorithm of Selecting more than Four Satellites from GNSS". In: International Conference on Advanced Computer Science and Electronics Information (ICACSEI 2013). 2013.

[9] Chan-Woo Park and Jonathan P. How. "METHOD AND APPARATUS FOR SELECTING OPTIMAL SATELITTES IN GLOBAL POSITIONING SYSTEM". Pat. US 6,727,850 B2. 2004.

[10] Masahiko Kihara and Tsuyoshi Okada. "A Satellite Selection Method and Accuracy for the Global Positioning System". In: NAVIGATION, Journal of the Institute of Navigation, Volume 31, Number 1. 1984.

[11] Nuria Blanco-Delgado and Fernando D. Nunes. "Satellite Selection Method for Multi-Constellation GNSS Using Convex Geometry". In: IEEE Transactions on Vehicular Technology 59.9 (Nov. 2010). ISSN: 1939-9359. DOI: $10.1109 /$ tvt . 2010. 2072939. URL: http://dx . doi . org/ 10 . $1109 /$ TVT.2010.2072939.
[12] Nuria Blanco-Delgado, Fernando D. Nunes, and Gonzalo Seco-Granados. "Relation between GDOP and the Geometry of the Satellite Constellation". In: 2011.

[13] Makarand S. Phatak. "Recursive Method for Optimum GPS Satellite Selection”. In: IEEE, 2001.

[14] J. Song, G. Xue, and Y. Kang. “A Novel Method for Optimum Global Positioning System Satellite Selection Based on a Modified Genetic Algorithm". In: PLoS ONE 11.3 (2016). DOI: 10.1371 / journal. pone. 0150005 .

[15] Dan Simon and Hossny El-Sherief. "Navigation satellite selection using neural networks". In: Neurocomputing 7.3 (1995), 247258. ISSN: 0925-2312. DOI: 10 . $1016 / 0925-2312$ (94) $00024-\mathrm{m}$. URL: http : / / dx .doi.org/10.1016/0925 2312 (94) $00024-\mathrm{M}$.

[16] Todd Walter and Per Enge. "Weighted RAIM for Precision Approach". In: Proceedins of the ION GPS. Institute of Navigation, 1995, pp. 1995 -2004.

[17] FAA GEAS Panel. GNSS Evolutionary Architecture Study: Phase I - Panel Report. Report. GNSS Evolutionary Architecture Study Panel, 2008.

[18] FAA GEAS Panel. Phase II of the GNSS Evolutionary Architecture Study. Report. GNSS Evolutionary Architecture Study Panel, Feb. 2010.

[19] Working Group C ARAIM Technical Subgroup. Interim Report Issue 1.0. Tech. rep. 2012. URL: http: / / www - gps . gov / policy / cooperation / europe/2015/working-group-c/.

[20] Working Group C ARAIM Technical Subgroup. Milestone 2 Report. Tech. rep. 2015. URL: http: / / www . gps . gov/policy / cooperation/europe / 2015 /working-group-c/.

[21] Daniel Gerbeth et al. "Optimized Selection of Satellite Subsets for a Multi-Constellation GBAS". In: Institute of Navigation International Technical Meeting 2016, ITM 2016. Vol. 1. Monterey, California, 2016, pp. 360-367.

[22] GPS Lab Stanford. MAAST for ARAIM Version 0.3. URL: http: / / waas.stanford.edu/staff/ maast/maast.html.

[23] Shau Shiun Jan et al. "Matlab Simulation Toolset for SBAS Availability Analysis". In: Proceedings of the 14th International Technical Meeting of the Satellite Division of The Institute of Navigation (ION GPS 2001). Salt Lake City, UT, 2001, pp. 2366-2375. 